\title{
Choreographed Matter
}

\author{
Olga Mesa \\ Roger Williams University and Harvard Graduate School of Design | United States | \\ omesa@rwu.edu | omesa@gsd.harvard.edu
}

\begin{abstract}
This research proposes an animation technique using external stimuli to activate kinematic transformations in material. Inherent material properties were enhanced to obtain specific behaviors. Carefully designed but easily fabricated two-dimensional paper constructions animate into three-dimensional configurations through water absorption in three different conditions: Surface, Datum, and Depth. Principles of origami, fiber orientation, and wax patterns are used to control movement in response to choreographic considerations. Digital workflows and analysis coupled with physical prototyping were used to produce patterns and informed a formal and performative taxonomy. Choreographed Matter contributes to expanding the expressive potential of material-based responsive systems and animation.
\end{abstract}

Keywords: Choreographed matter; Responsive materials; Animation techniques; Animated Origami; Smart materials.

\section{INTRODUCTION}

The word animation comes from the Latin "animare"- which means to give breath to or to instill with life, and a sign associated with living organisms is motion in response to external stimuli (Wilson, 1998). As such, creating the illusion of movement in animations is essential to convey that something is alive. Activating lifeless objects is not only a challenge for animators but also for designers from many disciplines, as the need for responsive and dynamic environments grows.

Current animation techniques use computational tools, digital fabrication, and have recently integrated the use of real physical materials for their textural and formal qualities. However, most overlook the inherent possibilities in material to achieve a dynamic response demonstrated by responsive material systems.

Responsive systems that are sensitive to user or environmental inputs are increasingly pervasive in the design disciplines. Among them, material-based systems present smart interactions where material moves in reaction to external stimuli. However, there is potential to develop further these systems for their formal dynamic qualities as tools of expression and, by doing so, offer new opportunities for their actuation and animation.

Instead of creating the illusion of movement, this research proposes an animation technique, Choreographed Matter, which uses external forces to trigger a kinetic transformation in a material by harnessing the properties of the material itself and further increasing its capabilities. By introducing water as an agent of transformation to carefully designed paper constructions, this research presents a method of animating material by producing precise and controllable movement. By determining the orientation of the paper's grain and printing wax ink patterns onto it, the paper is programmed to differential water absorption thereby actuating the material to extend or to swell and bend in a specifically designed direction. Using this technique, a fifteen-minute material animation was developed considering aspects of choreography (Figure 1). Kinematic principles were used in the design of folding patterns, to allow two-dimensional objects to transform into three-dimensional configurations and vice versa.

This research exploits the dynamic properties of materials for both their functional and expressive potential, thus contributing not only to material-based responsive systems that react to environmental inputs but also to expanding the possibilities of animation techniques.

\section{BACKGROUND}

There is a parallel between non-living responsive systems and the response inherent in living organisms whereby a body perceives an external stimulus, processes it in the brain, and sends information to the acting muscles (Wadhawan, 2007). Mediated by a type of sensor, a control, and an actuating mechanism, variations of this action-reaction relationship are found in animals, plants, and other entities that respond to the environment in which they exist. Often movement and transformation (outputs) are the response to a given stimulus (input).

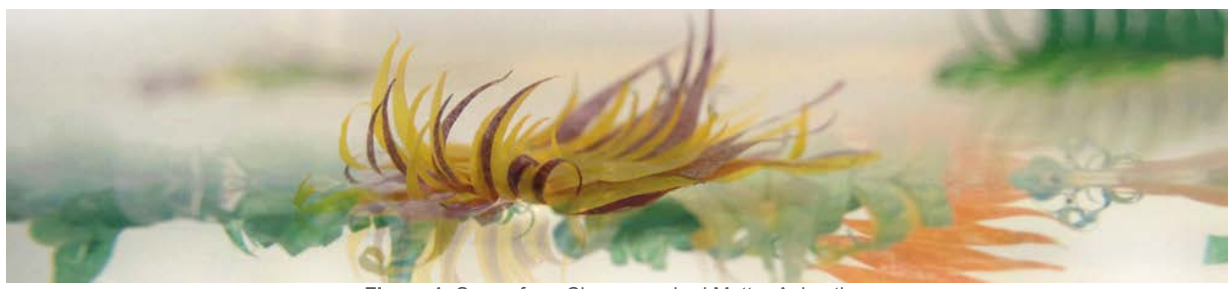

Figure 1: Scene from Choreographed Matter Animation. 


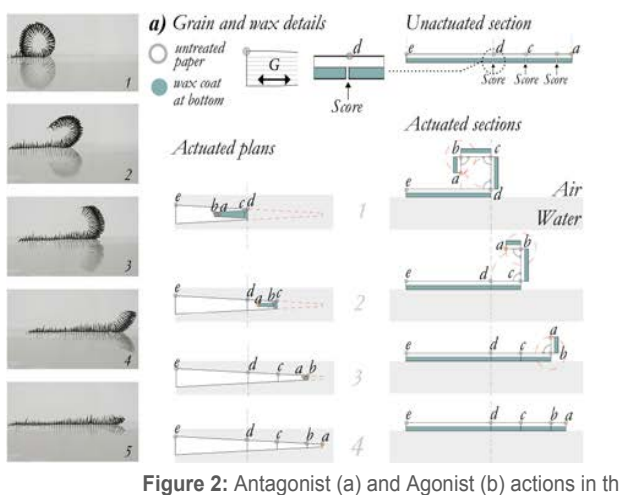

Figure 2: Antagonist (a) and Agonist (b) actions in the paper constructions actuated by water absorption

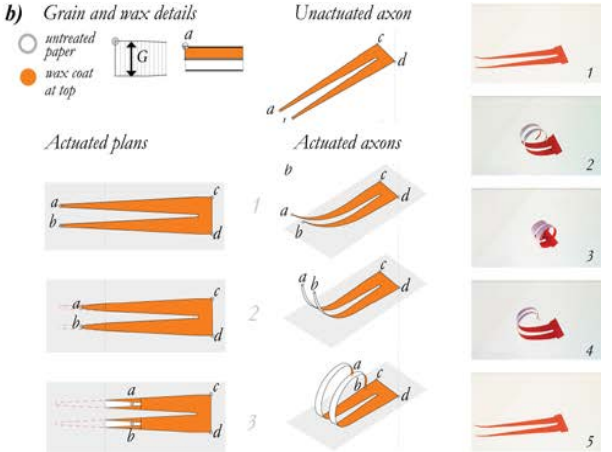

Designers and engineers have used electronic sensors, and mechanical hardware with the ambition to design systems that transform in reaction to external inputs. However, to overcome the expense, maintenance, and propensity to failure associated with these systems other alternatives that rely on material properties have developed in recent years (Menges and Reichert, 2012). Many of these initiatives are inspired by interactions found in natural systems. The work by the MIT Self-Assembly Lab on programmable materials (Tibbits, 2016) has successfully embedded carbon fiber, textiles, and wood filaments with sensorial and actuating capabilities (Tibbits, 2017). 'Hygromorph' and 'Hydroskin' by the Institute for Computational Design and Construction effectively demonstrate the anisotropic and hygroscopic qualities of wood veneer to open and close pores in response to moisture (Correa et al, 2013). Moreover, these research groups have collaborated to $4 d$ 3d-print hydrophilic polymers (Tibbits et al, 2014) and a combination of polymers and wood fiber filaments that expand over time when in contact with water (Correa et al, 2015 and Correa and Menges, 2015). Additionally, self-bending of singlecrystalline silicon films in reaction to water droplets have obtained shape-change transformations from $2 \mathrm{~d}$ to $3 \mathrm{~d}$ at the $\mathrm{mm}$ scale (Guo et al, 2009).

Found in nature, self-folding has sparked research examples of programmable matter in the science and design disciplines. To name a few, polystyrene sheets with black ink creases provide localized absorption of light, producing self-folding response (Liu et al, 2012), polyvinyl chloride laminated sheets based on metalized polyester film self-fold under heat exposure (Miyashita, 2014) and paper onto which origami patterns are printed with ink, dries into three-dimensional forms (Etherington, 2012). Shape memory origami laminates hinge in response to heat (Tolley et al, 2014), triangulated glass-reinforced sheets fold into predesigned, and reconfigurable shapes using shape memory actuators that react to joules-heat through electric current (Hawkes et al, 2010). These investigations employ principles of origami using composite materials supporting the connection between pleats and dynamic structures with some demonstrating the ability to be reversible and reconfigurable. These investigations range in scale, time response, fabrication complexity, and in most instances require highly specialized fabrication and intense computational methods for their design. Furthermore, they are rarely explored for the latent narrative potential and the expressive possibilities inherent in dynamic systems.

Regarding animations, designers are progressively using computer-generated techniques and more recently digital fabrication (Dear, 2013) to create the illusion of motion. Contemporary animated motion pictures like /lse of the Dog (Anderson et al, 2018), Fantastic Mr. Fox (Anderson et al, 2009), and many stop-motion animations that use origami folding show an increasing interest in reviving the incorporation of real materials. In addition, films like L'Écume des jours (Gondry, 2013) exquisitely feature animated food, exploring materials for their formal qualities (color, texture, etc). However, in these examples materials are not used for their potential to achieve movement and transformation as tools for expression.

Identifying opportunities from the literature review, the following goals were established:

- Design a geometry-material system that responds dynamically both predictively and quickly (factor of seconds) to an external stimulus.

- Propose an economical and simple to fabricate material-based animation technique at a scale that is suitable for recording transformation and that is accessible to a wide range of designers interested in investigating material-based transformations.

- Produce an animation using such technique and in doing so, explore the potential expressive and emotive content of dynamic responsive material systems.

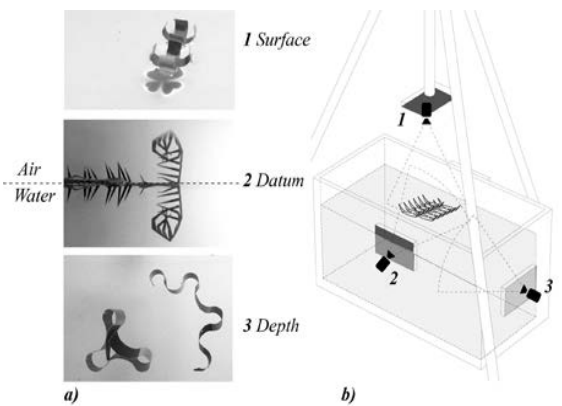

Figure 3: a) Animation Structure. b) Animation Recording Set-up. 


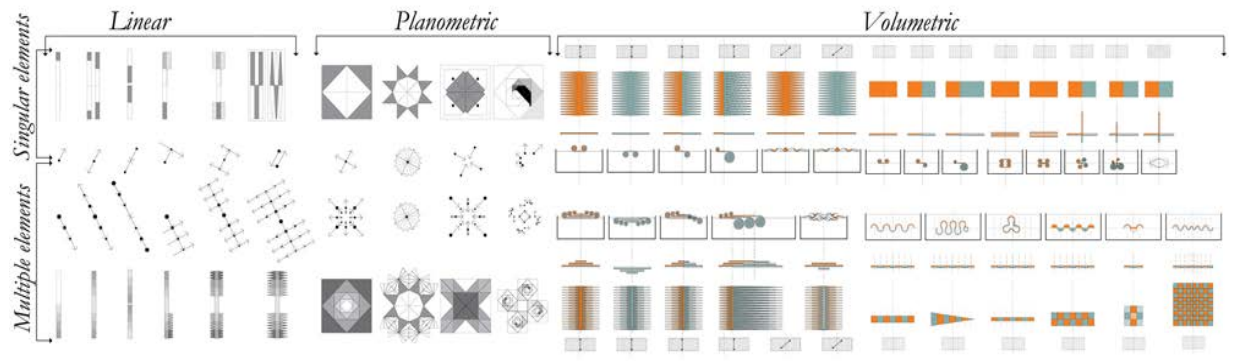

Figure 4: Formal and Performative Taxonomy

\section{METHODS}

The study of fundamental concepts of action | reaction and the analysis of relevant precedents were followed by identifying principles to design a responsive geo-material system and a methodology to implement in the production of the material-based animation (Figure 1).

A geo-material vocabulary was correlated to behavior and aspects of choreography and it was implemented in the fifteen-minute animation. To develop the vocabulary, digital workflows were employed to generate flat origami patterns that were physically tested by actuating them with water. Origami simulations helped analyze the relationship between motion and geometry to refine the final patterns featured in the animation. The physical prototypes were fabricated using a composite technique of paper and wax coatings and actuated using water in a tank.

\section{GEO-MATERIAL AND EXTERNAL STIMULUS INTERACTION}

Choreographed Matter studies the potential that arises when coupling a material's structural composition, its geometry, and an external stimulus to achieve a particular movement. Paper, a readily available material presenting inherent anisotropic qualities and non-linear mechanical behavior, was chosen as the material and water as the agent of transformation (Figure 2). Kinematic material affordances were identified and enhanced. By selectively masking areas of paper using wax (effectively changing absorption) and employing kinematic principles that translated into folds, the water was channeled in such a way that the material actuates and achieves the desired movement.

\section{ANIMATION STRUCTURE, TAXONOMY, AND CHOREOGRAPHY}

The animation was conceived as a sectional journey traveling from the surface of the water to its depth, passing through the line between air and water (Figure 3). This framework guided the development of the animated paper constructions. These three settings, Surface, Datum, and Depth, established the circumstances for actuation and the context for the performance of narrative. Choreographic considerations within each setting such as articulation of movement, timing, and use of space informed the patterns for the origami constructs. In the animation, the different perspectives related to these conditions were recorded using a Nikon D700 camera and the time to transform from initial to final stage was documented.

Two distinct actions were defined in the animated origami: Antagonist (extending) (Figure 2a). and Agonist (bending) (Figure 2b). Paper constructions presenting either one of these actions or combinations of both were tested to actuate in the three different settings relative to water and modifications were made taking advantage of the possibilities presented in each. A taxonomy related form to movement where motion direction was defined and movements from simple to complex and concatenated were translated into geometric patterns (Figure 4).

\section{ANALYSIS AND RESULTS}

Concerning the articulation of movement, the cellular structure of paper plays a key role in controlling the movement of the origami designs. The designs are activated by the absorption of water through capillary action; opening previously made paper creases, like hinges. Similar to traditional origami, the side onto which the score is made determines the opening direction (Figure
Side of score in relation to Different angles of scores

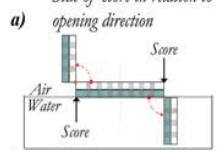

b) Score pattern to control timing of actuation

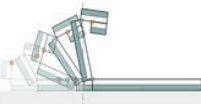

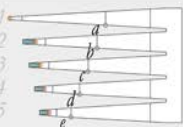
c)
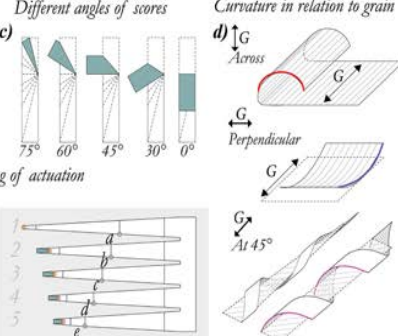

Wax placement in section e)

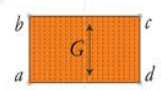

$a, b$ Sections

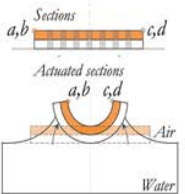

untrated paper 9 wax coat at top 1 wax coat at bottom f)

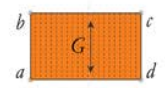

g)
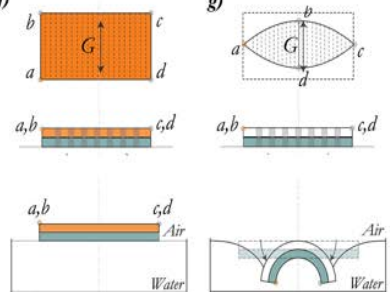

Figure 5: a), b) and c) Folding principles relative to actuation d) Curvature in relation to grain orientation e), f) and g) Wax placement in section relative to behavior 


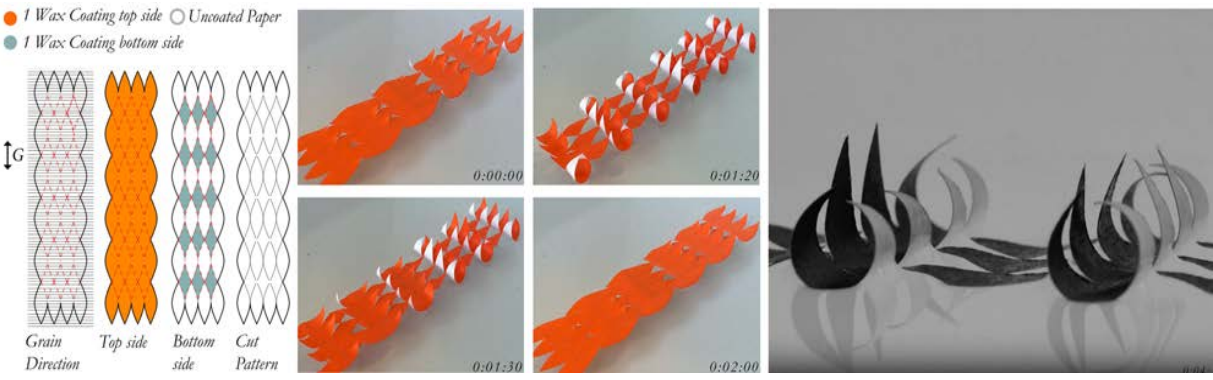

Figure 6: Surface with combination of grain orientation displaying anchoring and agonist actions

5a). Treating the paper with wax coatings to be selectively hydrophobic achieves different rates of absorption to obtain bending and buckling behaviors. Samples were produced to study the number of coatings and the side onto which they are applied to control water absorption. Based on these, wax is applied on both sides of the paper if the area is intended to be hydrophobic and remain flat (Figure $5 \mathrm{f}$ ). In contrast, wax is applied only on the front (Figure $5 \mathrm{e}$ ) or only on the backside (Figure $5 \mathrm{~g}$ ) to create differential swelling of paper and thus, induce buckling and bending upwards or downwards.

Additionally, the orientation of the paper grain is essential in controlling movement. The structural composition of the paper used is unidirectional, enabling it to expand, fold, and bend easily across its grain and less easily perpendicular to its grain. To determine the different flexing actions relative to fiber orientation, a paper test with a radial design was placed in water. As the water is absorbed, differences in the way of bending and spiraling were observed depending on the different orientation angles of the fiber (Figure 5d).

The origami designs obtained behavioral differences by employing the geometric principles mentioned above. Antagonist singular movements showing extension were achieved as well as sequential movements where a series of folds parallel to each other assures in-plane actuation giving the illusion of locomotion. Conversely, a change of direction is achieved by angling creases with respect to each other (Figure $5 \mathrm{c}$ ). Also, different degrees of bending were obtained depending on grain orientation. The bending behaviors were reversible while the extending ones were not. When combining different grain orientations and crease patterns in a single design more complex movements were achieved like bending in one axis while extending parts of the design perpendicular to that axis (Figure 4).
Timing was controlled geometrically and by adjusting the water temperature. Activating folds consecutively was controlled by spacing them in precise intervals relative to each other (Figure $5 \mathrm{~b}$ ). As water is absorbed, a greater distance between creases means a longer period for actuation and a shorter distance generates faster consecutive movements (Figure $5 b$ ). Some of the behaviors achieved were synchronized (Figure 9b) while others were designed to display chain reactions (Figure 10). By increasing the water temperature, thus reducing surface tension of the water and accelerating capillary action, the activation speed of the origami designs is increased. In the animation, adjusting the water temperature was used to speed up some movements or to slow them down.

The use of space in the three different settings informed the design of the constructions. Some constructs were planned to actuate as singular objects or in a linear fashion while others transformed in the $\mathrm{x}$ and $\mathrm{y}$-axis and others engaged the $x, y$, and $z$-axis as they moved (Figure 4). Some designs self-assembled when actuated (Figures 7 and 8).

Lastly, what was at first conceived as the agent of transformation became the context for actuation and narrative. As such, as the animated origami actuated, the different settings of water presented unique opportunities for expression. For instance, in the Surface setting, the fluidity of the surface registered the movement of the paper constructs (Figure 8). In Datum, internal reflection brought possibilities for animated reflective symmetries (Figure 9) and Depth presented an environment where objects appear floating while animated (Figure 12).
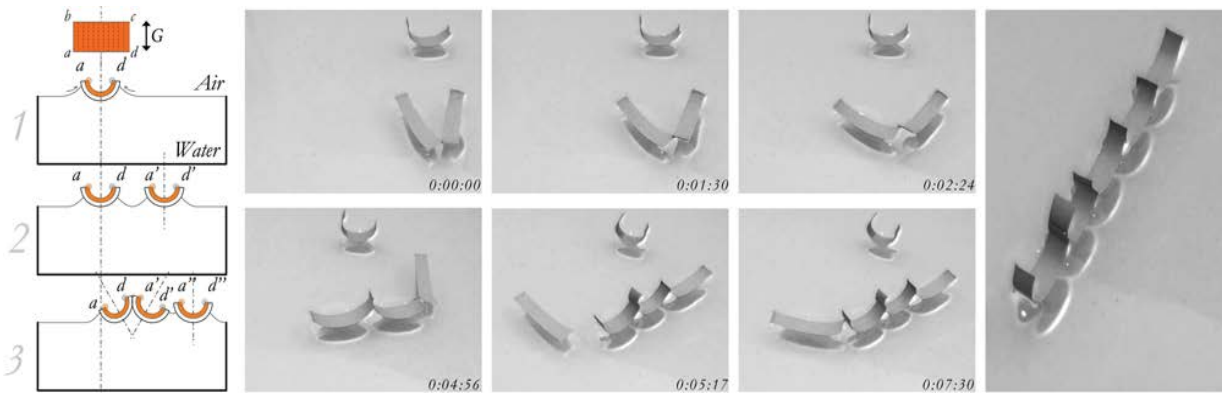

Figure 7: Self-assembled chain configuration and its caustic projection 

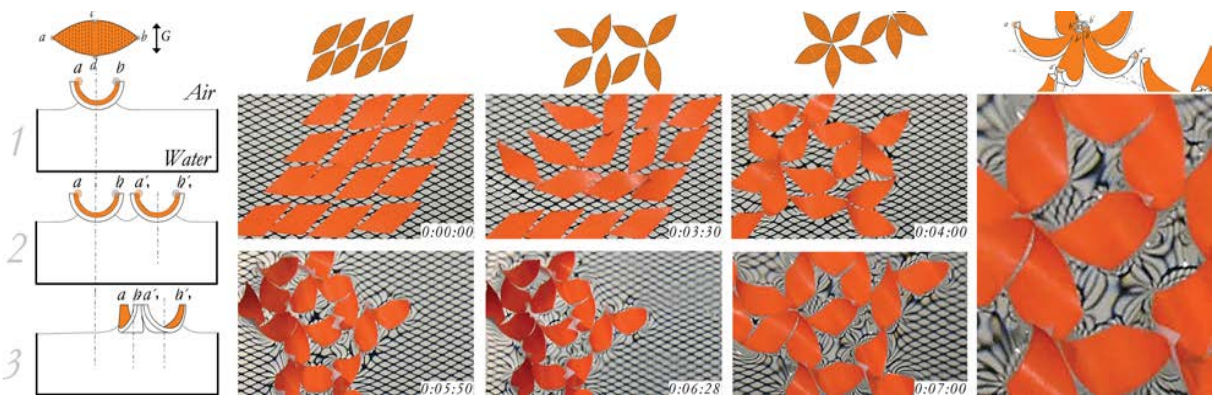

Figure 8: Self-assembled configuration showing lattice distortions

\section{SURFACE}

Referring to the animated origami designed for the Surface setting, the surface tension of the water provides an anchoring ground for the faces of the paper that are in contact with it. This allows the paper to unfold, curl, or both and move without sinking. By absorbing water, Antagonists designs unfold pushing on the surface of the water. In some cases, portions of the designs actuate through capillary action transforming from $2 \mathrm{~d}$ to $3 \mathrm{~d}$ without touching the surface of the water.

Regarding the Agonist origami, the paper designs curl as water is absorbed. Shape-change from a flat stage to a concave form after bending upwards is achieved if the waxtreated side is facing up and the side facing down is in contact with the water. Contrary to the Antagonist behavior, a return to the initial flat stage happens in a factor of seconds displaying reversibility (Figure $2 \mathrm{~b}$ ). The motion is not as repeatable until the paper construction is dry.

More complex designs show the activation of some areas while not others (Figure 6). This was achieved by applying wax on both sides for anchoring elements and applying wax only on the front to enable curling and relaxation. As adhesion forces between the water molecules and the paper are greater than the cohesion forces between water molecules, a meniscus forms along the sides of the transforming Agonist origami, thus pulling the surface of the water (Figure $5 \mathrm{e}$ and 6).

Temporal self-assembled configurations were achieved in the Agonist origami and controlled by the boundary of the flat patterns. As the designs bend into their threedimensional configurations, they attract each other forming self-assembled configurations that dissipate as the designs relax back into their flat state (Figure 7). Figure 7 shows a linear chain assembly and Figure 8, one that approximates a pentagonal formation. Chain reaction behaviors were obtained by combining flexing and contracting designs. In the surface condition, the movements have the quality of being cushioned as the origami actuates pushing and pulling on the water (Figures 6, 7, and 8). The water surface becomes a dynamic canvas for visualizing transformation. In the animation, this effect was amplified by projecting images of regular lattices onto the water surface. As the origami moves, distortions of the lattices are seen (Figure 8). Also as light is shone through the modified surface, visual imagery projects onto the bottom of the tank due to caustic phenomena (Figures 3 and 7).

\section{DATUM}

At the Datum, the same principles described above apply but at this setting the phenomenon of total internal reflection provides a mirror-like surface creating animated visual symmetries (Figure 9). Due to this, the objects above the water level are concealed and only the movement below the water level is seen and reflected upwards (Figure 9a). A movement from flat to convex is achieved if the wax-treated side is facing down and the side facing upwards absorbs water. Therefore, by tapering the boundary of the flat patterns, these can puncture the surface of the water as they actuate, thereby enabling bending downwards towards the water (Figure $5 \mathrm{~g}$ ). Four $\mathrm{mm}$ diameter rolling was achieved (Figure 9c). For the tight rolling, $5 \mathrm{~cm}$ long $60 \mathrm{gsm}$ thick paper was used and the wax pattern was applied in the same patterns but with fewer coatings than for the $90 \mathrm{gsm}$ thick paper. Spiraling actions were achieved (Figure 9b). The actuation of consecutive folds was possible by nesting layers of treated paper, a)

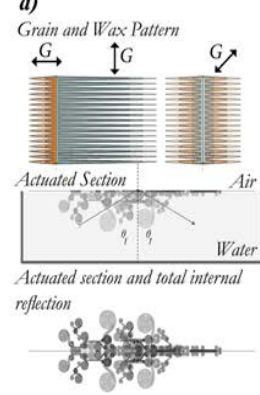

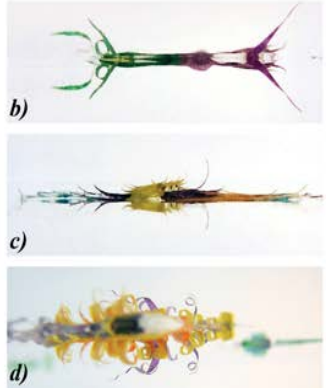
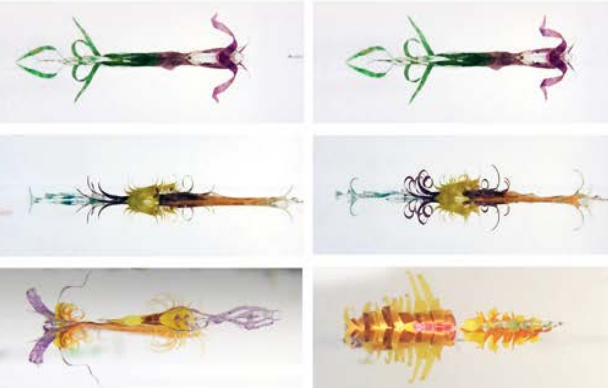

Figure 9: Paper constructions actuated at the Datum setting showing total internal reflection 


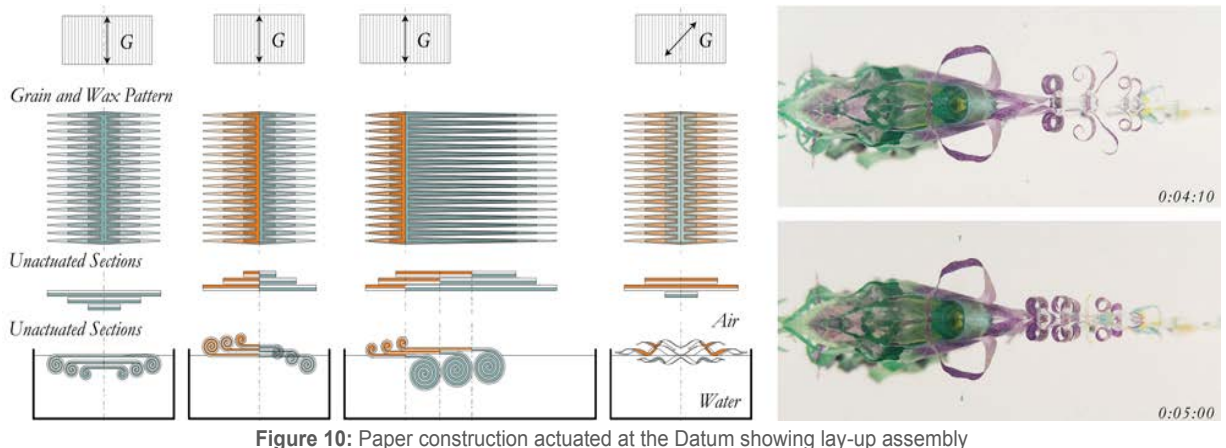

controlling water absorption sequentially where the outer layers actuate before the inner layers of the design (Figures 9c, 9d, and 10).

\section{DEPTH}

In the Depth setting, similar principles to the ones mentioned above were applied, but the movements occur underwater. This enables the possibility of seeing transformations from both sides of the designs. By applying wax on the front and back sides of the paper in an alternating pattern and accounting for the direction of the grain, rolling, and sinuous configurations were obtained (Figure 11). The geometric pattern and the treatment of paper dictated the frequency, amplitude, and the line where wave direction happens. This enabled control of the spring behavior in the different designs. Figure 11 shows corrugations that reorient by trapping air bubbles, giving the designs a buoyant quality. Figure 12 shows a design that features both extending and bending behaviors. This design starts as a flat strip that corrugates, then it flips as a previously made score hinges upon activation and it corrugates into a three-dimensional triangular form that closes on itself.

\section{DIGITAL ANALYSIS}

Physical prototyping as well as a digital workflow using Rhino, Grasshopper, and Origamizer were employed to generate two-dimensional patterns and simulate the behavior of the animated designs. For the Antagonist actions, two-dimensional patterns were imported in Origamizer to simulate unfolding (Figure 13a and 13b). Their actions matched with the physical prototypes. Although, in the physical prototypes, the reversible behavior was not yet obtained.
Finite Element Analysis simulations were employed using Abaqus to simulate the behavior of Agonist actions. To simulate swelling of paper, a heat expansion coefficient was assigned to the areas intended not to have wax. An increase in temperature on those surfaces emulates swelling of those areas. Figure $13 \mathrm{c}$ shows an increase of $20 \%$ to simulate the expansion ratio relative to the absorption rate.

Many of the designs show a combination of both actions (unfolding and swelling that causes bending). Therefore, developing a workflow to simulate their combined behavior would be beneficial. However, for this research more consideration was given to the generation of patterns and grain orientations that were obtained through physical iteration and modeling using Rhino and Grasshopper.

\section{FABRICATION TECHNIQUE}

The fabrication technique involves drawing the geometry of the designs in their flat stage through a Rhino/Grasshopper workflow taking into account orientation relative to the paper grain and determining the areas where the wax pattern will be applied to make the material selectively more or less hydrophobic.

The designs are then printed onto $90 \mathrm{gsm}$ thick bond paper or $60 \mathrm{gsm}$ thick tracing paper and the wax pattern is deposited using a Xerox ColorQube printer. Registrations are used to assure alignment when depositing wax coatings on both the top and the bottom sides of a design. Designs are simply laser cut using a Universal 150W laser cutter and placed in a tank of water to transform from their flat stages to three-dimensional configurations and vice versa (Figure14).
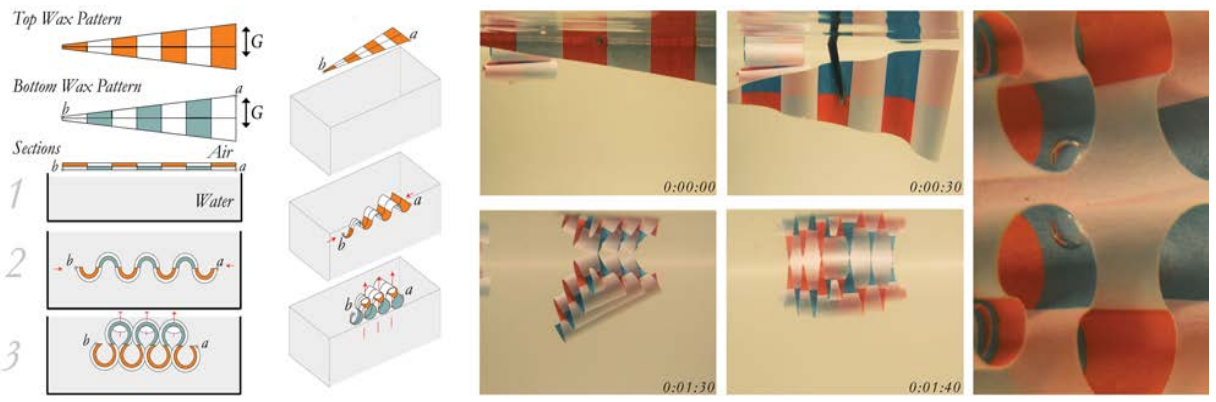

Figure 11: Three-dimensional corrugation with triangular boundary actuated underwater 

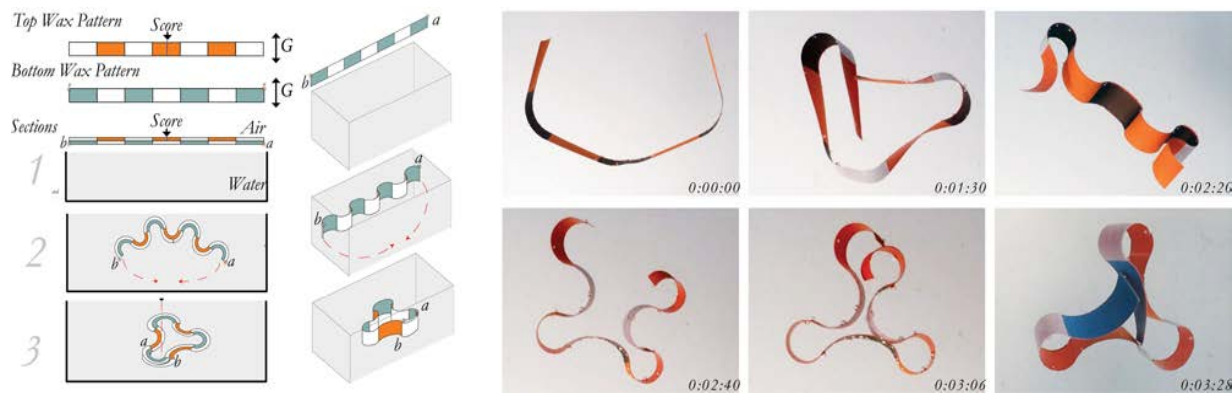

Figure 12: Three-dimensional corrugation with rectangular boundary actuated underwater

\section{CONCLUSIONS}

In regards to the goals stated at the start of this research, a geometry-material system was designed to achieve dynamic transformations in response to external stimuli. Designed paper constructions actuate when in contact with water to produce controllable behaviors in a factor of seconds. The type of movement was predicted and controlled by applying principles of folded geometry and by enhancing the inherent anisotropic capacities of paper. To generate a specific behavior, the fiber orientation of paper was determined and wax patterns were selectively applied to tune the material's reaction to water absorption. As water is absorbed, differential swelling of the paper and opening of previously made folds occur resulting in predictable behaviors such as bending, extending, and spiraling. A combination of different fiber orientations and wax applications were used to achieve more complex movements. A formal and performative taxonomy was produced relating geometry and material's designed composition, to behavior.

The research also aimed to propose a way of animating material that would be accessible to a wide range of designers interested in responsive systems. For this purpose, the proposed technique involves a readily available material that has inherent anisotropic and hydrophilic characteristics (paper), a commercially available wax-ink printer (Xerox ColorQube), and a tank of water to actuate the animated paper constructions. These choices make prototyping ease to iterate and economical to produce at the centimeter scale, thus enabling recording of transformations viable with a standard camera.
The fifteen-minute animation produced using the Choreographed Matter technique was both a proof of concept and a medium to investigate possible expressive characteristics of material-based responsive systems. Through the development of this animation, design limitations and possibilities were identified placing demands on the development of the technique itself. The defined sectional framework of the animation included three different settings: Surface, Datum, and Depth, which provided rich conditions for actuation and the exploration of narrative.

Choreographic aspects informed the design of the paper constructions. The material's composition and the geometric patterns were designed for the articulation of specific movement. A vocabulary of behaviors was developed with the aspiration to communicate emotive content. Extending, contracting, curling, and spiraling actions were exploited for their expressive potential in the animation. Timing was controlled by either the geometric arrangement -as this set the sequence of actuation- or by modifying the temperature of the water to accelerate or slow down movements. The paper constructions took into account the use of space as they were to be animated with a range of designs from simple and linear to complex and volumetric when actuated to their final stages.

Achieving synchronization, chain reaction, and selfassembling demonstrate targeted use of geometry and material treatment as well as an understanding of the external stimuli to achieve nuances in the articulation of movement where timing and use of space were considered. The interactions designed in Choreographed Matter are more complex and intertwined than anticipated.
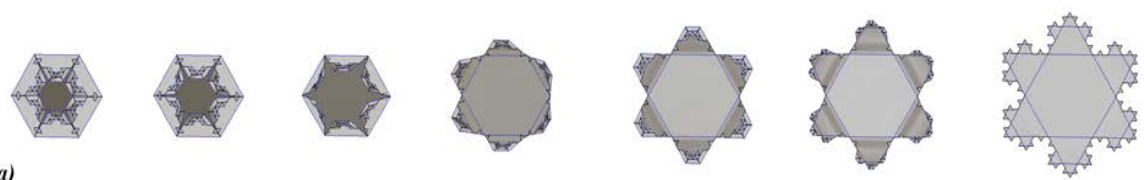

a)
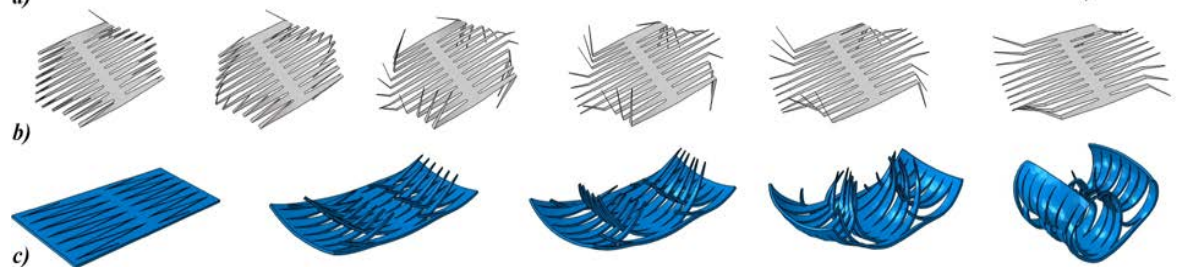

Figure 13: a) Origamizer Simulation b) Origamizer Simulation showing sequential folds c) FEA Simulation of Agonist paper constuction 


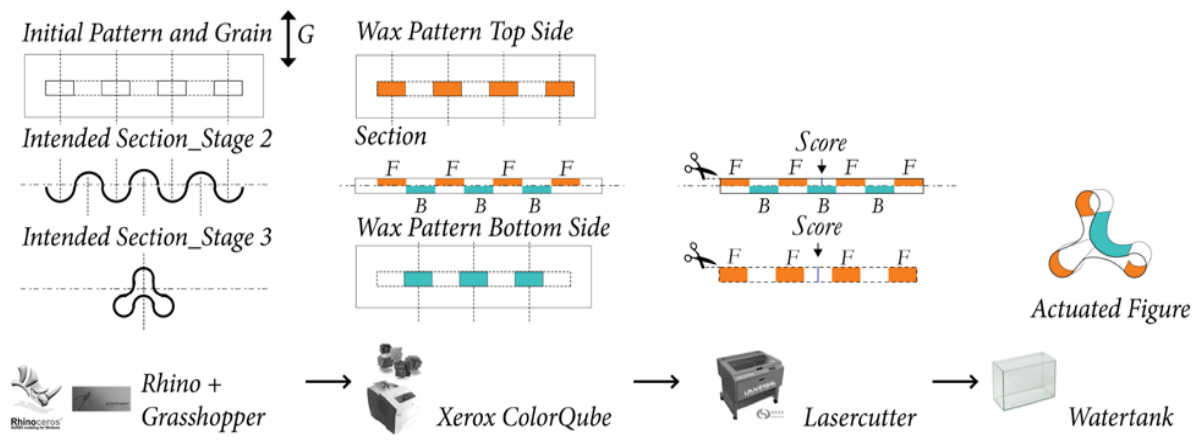

Figure 14: Fabrication Workflow

For instance, the source of actuation (water) became a ground to register the motion of the animated pleats. This opened an array of possibilities for formal and dynamic expression. The process involved control and unpredictability as well as empiricisms and scientific analysis to be in constant dialogue. This research offers possibilities for material-based responsive systems to be more expressive, to awaken our imagination, and to celebrate the alternative ways in which we respond to the ever-changing environment around us.

\section{ACKNOWLEDGMENTS}

I would like to dedicate this work to my family: Olga Higuera, Claudia Mesa, Natalia Mesa, and Aaron Brode. Their caring support and critical insight were instrumental in the development of this research. Thank you to Natalia Mesa because her knowledge of dance inspired several designs. Thank you to Pico Velasquez, for her wonderful support and to her, Alicia Millet and Kate Cusik for their assistance in preparing the preliminary diagrams, to Johannes Bass for his aid with the FEA simulations, and to Josh Gosh for allowing me access to a Xerox ColorQube. Finally, thank you to Allen Sayegh and Panagiotis Michelatos, for their thoughtful input and guidance .O.

\section{REFERENCES}

Dawson, J. (Producers), Anderson, W., Baumbach, N., Clooney, G., Streep, M., Schwartzman, J., \& Murray, B. (Writers). (2009). Fantastic Mr. Fox [Motion picture]. United States: 20th Century Fox.

Anderson, W. (Director), Anderson, W., Rudin, S., Rales, S. \& Dawson, J. (Producers), Anderson, W., Coppola, R., Schwartzman, J., Nomura, K., (Writers). (2018). Isle of dogs [Motion picture]. United States \& Germany: 20th Century Fox.

Correa, D., Krieg, O. D., Menges, A., Reichert, S., \& Rinderspacher, K. (2013). Hygroskin: A climate-responsive prototype project based on the elastic and hygroscopic properties of wood. In ACADIA 2013: Adaptive Architecture Proceedings of the 33rd Annual Conference of the Association for Computer-Aided Design in Architecture (pp. 33-42). ACADIA.

Correa, D., Papadopoulou, A., Guberan, C., Jhaveri, N., Reichert, S., Menges, A., \& Tibbits, S. (2015). 3D-Printed Wood: Programming Hygroscopic Material Transformations. 3D Printing and Additive Manufacturing, 2(3), 106-116. https://doi.org/10.1089/3dp.2015.0022

Correa, David, and Achim Menges. 2015. "3D Printed Hygroscopic Programmable Material Systems." MRS Proceedings 1800: 2134303. https://doi.org/10.1557/opl.2015.644
Dear, E. \& Geleyn, Y. (Directors), Yates, B., Bretton, J. \& Gallagher, J.(Producers), W., Coppola, R., Schwartzman, J., Nomura, K., (Writers). (2013). The Bear and the Hare [Motion picture]. United States: Disney Studios.

Etherington, R. (2012, April 13). Hydro-Fold by Christophe Guberan. Retrieved from https://www.dezeen.com/2012/04/13/hydro-fold-bychristophe-guberan/

Gondry, M. (Director), Bossi, L., (Producers), Bossi, L. \& Gondry, M. (Writers). (2013). L'Écume des jours [Film]. France \& Belgium: Brio Films.

Guo, X., Li, H., Ahn, B. Y., Duoss, E. B., Hsia, K. J., Lewis, J. A., \& Nuzzo, R. G. (2009). Two- and three-dimensional folding of thin-film single-crystalline silicon for photovoltaic power applications. Proceedings of the National Academy of Sciences of the United States of America - PNAS, 106(48), 20149-20154. Print

Hawkes, E., An, B., Benbernou, N. M., Tanaka, H., Kim, S., Demaine, E. D., Rus, D., Wood, R. J. (2010). Programmable matter by folding. Proceedings of the National Academy of Sciences of the United States of America, 107(28), 1244112445.

Liu, Y., Boyles, J. K., Genzer, J., \& Dickey, M. D. (2012). Selffolding of polymer sheets using local light absorption. Soft Matter, 8(6), 1764-1769.

Menges, Achim, \& Reichert, Steffen. (2012). Material Capacity: Embedded Responsiveness. Architectural Design, 82(2), 5259 .

Miyashita, S., Meeker, L., Tolley, M. T., Wood, R. J., \& Rus, D. (2014). Self-folding miniature elastic electric devices. Smart Materials and Structures, 23(9), 94005.

Tibbits, S. (2014). 4D printing: Multi-material shape change. Architectural Design, 84(1), 116-121.

Tibbits, S. (2017). Active Matter. Cambridge: MIT Press.

Tibbits, S. (2016). Self-Assembly Lab. Self-Assembly Lab. Routledge.

Tolley, M. T., Felton, S. M., Miyashita, S., Aukes, D., Rus, D., \& Wood, R. J. (2014). Self-folding origami: Shape memory composites activated by uniform heating. Smart Materials and Structures, 23(9), 094006.

Wadhawan, V. (2007). Smart structures: Blurring the distinction between the living and the nonliving (Monographs on the physics and chemistry of materials; 65). Oxford; New York: Oxford University Press.

Wilson, F. (1998). The hand: How its use shapes the brain, language, and human culture (1st ed.). New York: Pantheon Books. 